\title{
Electrophysiological Evidence That the Retrosplenial Cortex Displays a Strong and Specific Activation Phased with Hippocampal Theta during Paradoxical (REM) Sleep
}

\author{
-Bruna Del Vechio Koike, ${ }^{1,2 *}$ - Kelly Soares Farias, ${ }^{1,2 *}$-Francesca Billwiller, ${ }^{2 *}$-Daniel Almeida-Filho, ${ }^{1}$ \\ (D)Paul-Antoine Libourel, ${ }^{2}$ Alix Tiran-Cappello, ${ }^{2}$ - Régis Parmentier, $^{2}$ ○Wilfredo Blanco, ${ }^{3}$ Sidarta Ribeiro, ${ }^{1}$ \\ (DPierre-Herve Luppi, ${ }^{2 *}$ and ${ }^{-C}$ Claudio Marcos Queiroz ${ }^{1 *}$ \\ ${ }^{1}$ Brain Institute, Federal University of Rio Grande do Norte, Natal, RN, 59056-450, Brazil, ${ }^{2}$ UMR 5292 CNRS/U1028 INSERM, Center of Research in \\ Neuroscience of Lyon, SLEEP Team, Université Claude Bernard Lyon I, Faculté de Médecine RTH Laennec, 69372 Lyon Cedex 08, France, and ${ }^{3}$ Computer \\ Science Department, State University of Rio Grande do Norte, Natal, RN 59072-970 Brazil
}

It is widely accepted that cortical neurons are similarly more activated during waking and paradoxical sleep (PS; aka REM) than during slow-wave sleep (SWS). However, we recently reported using Fos labeling that only a few limbic cortical structures including the retrosplenial cortex (RSC) and anterior cingulate cortex (ACA) contain a large number of neurons activated during PS hypersomnia. Our aim in the present study was to record local field potentials and unit activity from these two structures across all vigilance states in freely moving male rats to determine whether the RSC and the ACA are electrophysiologically specifically active during basal PS episodes. We found that theta power was significantly higher during PS than during active waking (aWK) similarly in the RSC and hippocampus (HPC) but not in ACA. Phase-amplitude coupling between HPC theta and gamma oscillations strongly and specifically increased in RSC during PS compared with aWK. It did not occur in ACA. Further, $68 \%$ and $43 \%$ of the units recorded in the RSC and ACA were significantly more active during PS than during aWK and SWS, respectively. In addition, neuronal discharge of RSC but not of ACA neurons increased just after the peak of hippocampal theta wave. Our results show for the first time that RSC neurons display enhanced spiking in synchrony with theta specifically during PS. We propose that activation of RSC neurons specifically during PS may play a role in the offline consolidation of spatial memories, and in the generation of vivid perceptual scenery during dreaming.

Key words: anterior cingulate cortex; gamma; hippocampus; retrosplenial cortex; sleep; theta

Significance Statement

Fifty years ago, Michel Jouvet used the term paradoxical to define REM sleep because of the simultaneous occurrence of a cortical activation similar to waking accompanied by muscle atonia. However, we recently demonstrated using functional neuroanatomy that only a few limbic structures including the retrosplenial cortex (RSC) and anterior cingulate cortex (ACA) are activated during PS. In the present study, we show for the first time that the RSC and ACA contain neurons firing more during PS than in any other state. Further, RSC neurons are firing in phase with the hippocampal theta rhythm. These data indicate that the RSC is very active during PS and could play a key role in memory consolidation taking place during this state.

\section{Introduction}

Paradoxical sleep (PS) is characterized by muscle atonia, rapid eye movement (REM), and cortical and hippocampal activation

\footnotetext{
Received Dec. 27, 2016; revised July 5, 2017; accepted July 11, 2017.

Author contributions: P.-H.L. and C.M.Q. designed research; B.D.V.K., K.S.F., F.B., A.T.-C., and R.P. performed research; B.D.V.K., K.S.F., D.A.-F., P.-A.L., W.B., and C.M.Q. analyzed data; S.R., P.-H.L., and C.M.Q. wrote the paper.

This work was supported by CNRS, Fondation pour la Recherche Médicale, Societé Francaise de Recherche et Médecine du Sommeil, University Claude Bernard of Lyon, CAPES/COFECUB (783/13) and Conselho Nacional de Desenvolvimento Científico e Tecnológico Grants 480875/2012-0 and 308775/2015-5 and, Fundação de Amparo à Pesquisa do Estado de São Paulo Grant 2013/07699-0 Center for Neuromathematics. We thank Diego Laplagne for insightful discussions and help with Wiener-Granger causality.
}

(Jouvet and Michel, 1959). Spectral analysis of electroencephalogram (EEG) and local field potential (LFP) recordings have shown that theta and gamma oscillations are preeminent in the prefrontal, somatosensory and parietal cortices and in the hippocampus (HPC) both during PS and waking (WK) but not dur-

*B.D.V.K., K.S.F., F.B., P.-H.L., and C.M.Q. contributed equally to this work.

The authors declare no competing financial interests.

Correspondence should be addressed to Dr. Pierre-Hervé Luppi, Team SLEEP,UMR 5292 CNRS/U1028 INSERM, 7 , Rue Guillaume Paradin, 69372 Lyon Cedex 08, France. E-mail: luppi@sommeil.univ-lyon1.fr. DOI:10.1523/JNEUROSCI.0026-17.2017

Copyright $\odot 2017$ the authors $\quad 0270-6474 / 17 / 378003-11 \$ 15.00 / 0$ 
ing slow-wave sleep (SWS; Maloney et al., 1997; Montgomery et al., 2008; Sirota et al., 2008; Scheffzük et al., 2011). Unit recordings confirmed that the discharge rate of neocortical neurons is higher both during PS and WK than during SWS (Steriade et al., 2001; Vyazovskiy et al., 2009). Further, it has been shown that part of the neurons located in the somatosensory and prefrontal cortices synchronize their firing with theta and gamma during PS (Sirota et al., 2008). On the other hand, we recently showed that the anterior cingulate cortex (ACA), retrosplenial cortex (RSC), and medial entorhinal cortex as well as the dentate gyrus of the HPC contain a large number of Fos-labeled neurons after PS hypersomnia (Renouard et al., 2015). These results suggest that these four limbic cortical structures are the only cortical structures containing a large number of neurons specifically activated during PS. However, a direct electrophysiological demonstration that neurons of these structures are highly active specifically during basal PS episodes was still lacking. Further, the relationship between the pattern of discharge of these neurons with that of hippocampal and local oscillations remained to be determined. In this context, we extracellularly recorded action potentials and LFPs from two of these neocortical areas (RSC and ACA) during naturally occurring sleep-wake states and correlated firing activities with hippocampal theta activity.

\section{Materials and Methods}

Animals. Male Sprague-Dawley rats (3-4 months old, 250-300 g, $n=12$, RRID:RGD_5508397) were supplied by Javier Labs and Charles River Laboratories and upon arrival, individually housed in controlled environment (temperature $21 \pm 1{ }^{\circ} \mathrm{C}$ and humidity at $75 \%$ ) under a $12 \mathrm{~h}$ light/dark cycle (lights on 07:00 A.M.). Standard rodent food and water were available ad libitum throughout the experiments. Experimental protocols were approved by the institutional animal care and use committee of the University of Lyon 1 and the French Ministère de l'Enseignement Supérieur et de la Recherche and were in accordance with the French and European Community guidelines for the use of animals in research as well as the Ethical Committee on Animal Use of the Federal University of Rio Grande do Norte, Brazil (no. 025/2014).

Multielectrode implantation. Electrophysiological signals were obtained using customized multielectrode arrays chronically implanted under isoflurane anesthesia (induction: 5\%; maintenance: 1.5-2\%). Each array consisted of $8-32$ tungsten microwires covered with polyamide ( 35 $\mu \mathrm{m}$ diameter; with $\sim 100-350 \mathrm{k} \Omega$ impedance; California Fine Wire) and spaced by $200-250 \mu \mathrm{m}$ in a rectangular-shaped arrangement. A total of 60 deep electrodes were stereotaxically placed in the dorsal HPC (AP: 3.0-4.4; DV: 3.8; ML: 1.1-2.6, in mm), ACA (AP: 0.5-2.0; DV: -3.1; ML: 0.1-1.6 mm), and RSC (AP: 6.5-8.0; DV: 2.2; ML: 1.2-2.9; angle: $13^{\circ}$; Paxinos and Watson, 1997). Animals were also implanted with two stainless steel screws over the right frontal and left parietal cortices for monitoring electrocorticographic activity (ECoG). Two additional spherical electrodes were placed in the nuchal muscles for recording the EMG. An additional screw was placed over the cerebellum and served as a common reference for all electrodes. Arrays were fixed to the skull with acrylic cement using extra screws as anchors. In some animals (6 of 12), RSC arrays were fixed to a nanodrive (Cambridge NeuroTech), allowing dorsoventral movements. After surgery, rats received a nonsteroidal anti-inflammatory drug ( $5 \mathrm{mg} / \mathrm{kg}$ carprofen, i.p.) and antibiotics to attenuate pain and reduce infection. The animals were allowed to recover at least $7 \mathrm{~d}$ after surgery, with constant monitoring of the general behavior and body weight to monitor their health.

Electrophysiological recordings. After the recovery period, the animals were handled for $3 \mathrm{~d}$ before the beginning of the experiments to habituate them to the recording chamber. After 7-10 d, they were briefly anesthetized with isoflurane and connected to the recording system. The recordings began after a $1-2 \mathrm{~h}$ interval to allow the wash out of the anesthetics. Power spectrum analyses were compared for all states during the first and the last hours of recordings to verify that there was no residual effect of the anesthetic on EEG and LFP activities. The headstage was connected to a shielded cable attached to a motorized swivel to allow free movement of the animal within the recording chamber. Continuous electrophysiological recordings were made throughout the experiment. We focused the analysis on the period between 10:00 A.M. and 6:00 P.M. Electrophysiological recordings were performed using a multichannel acquisition processor (MAP System, Plexon). Signals were preamplified (VLSI headstages, $20 \times$ gain, Plexon), filtered (LFP: $0.1-500 \mathrm{~Hz}$ and spike waveforms: $0.3-40 \mathrm{kHz}$ ), amplified (20-100×), and digitalized (LFP: $2 \mathrm{kHz}$; multiunit activity: $40 \mathrm{kHz}$ ). Spike waveforms (56 points, $1.4 \mathrm{~ms}$ ) and their corresponding timestamps, as well as continuous LFP, were recorded continuously.

Data analysis. All LFP and spike data were analyzed using customized scripts running in MATLAB (MathWorks, RRID:SCR_001622). Five second windows of synchronous LFP, ECoG, and EMG data were used to score vigilance states into five different states: active wake (aWK), quiet wake (qWK), SWS, intermediate sleep (IS), and PS, according to classical criteria (Maloney et al., 1997; Boissard et al., 2002). The aWK state was differentiated from qWK by the presence of theta oscillation and movements (i.e., large amplitude, nonstationary EMG). Ambulation and exploration of the cage were observed during aWK but not during qWK. To remove from aWK sampling, microarousals occurring in between SWS or in between PS and SWS episodes, we included in our analysis only aWK episodes lasting at least $15 \mathrm{~s}$. The same criterion was applied to SWS and PS episodes to exclude transitional periods. Channels containing putative waveforms identified online were manually processed off-line for spike waveform separation and classification, when appropriate, using Offline Sorter v3 (Plexon, RRID:SCR_000012). Spike waveform parameters, such as trough-to-peak latency and peak asymmetry index were used to classify narrow and wide spikes (Sirota et al., 2008). Only units recorded in channels located within the target structure and with stable waveforms across the entire recording session were considered for further analysis (Fig. 4D). For each unit, we computed the mean firing rate and the mean interspike interval (ISI) during each vigilance state for the entire recording session. Such approach allowed the identification of firing patterns across aWK, SWS, and PS. ANOVA and paired $t$ test were used to determine whether firing rates of each neuron was statistically different between the three main states investigated (aWK, SWS, and PS). Power spectral density for ECoG and LFP signals were calculated using Welch's method ( $p$ welch function) for each $5 \mathrm{~s}$ period scored previously. For each period, normalization of the power spectrum was obtained by dividing the absolute power of each frequency value by the total power. Mean relative power was obtaining by averaging all $5 \mathrm{~s}$ periods for each state for each animal. Grand averages were calculated for all animals and for each state. For statistical analysis, relative power integrated over different frequency bands (delta: $0.5-4 \mathrm{~Hz}$, theta: $4-12 \mathrm{~Hz}$, gamma: $40-100 \mathrm{~Hz}$, and fast gamma $100-160 \mathrm{~Hz}$ ) was calculated. Hilbert-transformation (hilbert function) of the filtered (see below) frequency bands was used to extract the instantaneous phase and to determine the preferred phase of unit activity.

Granger causality analysis. To characterize directionality and strength of information between the hippocampus and the retrosplenial cortex during PS and aWK, Wiener-Granger causality (WGC) statistics were performed (Bressler and Seth, 2011). Such test calculates to which extent one time series improves the prediction of another time series compared with the prediction obtained using its own past values alone. Granger causality was computed between pairs of previously selected HPC and RSC LFP signals using the MVGC toolbox (Barnett and Seth, 2014). Such approach to WGC uses advanced vector autoregressive model theory to enhance accuracy and to facilitate the computation of causality in the frequency domain. First, raw, simultaneously recorded LFPs from the HPC and RSC were decimated to $200 \mathrm{~Hz}$. No LFP filtering was performed because it may hazard causalities estimates (Bressler and Seth, 2011). Concatenate aWK and PS episodes were analyzed separately. Model order was initially based on the cross-correlation peak-to-peak interval and heuristically set to 40. Although higher values did not improve WGC calculations (i.e., yielded similar results), it dramatically increased computational time. For each animal, statistical significance of the Granger causality in the frequency domain was assessed by time- 
shifting one LFP beyond the analytic window (between 1 and $2 \mathrm{~s}$ uniformly distributed lags). WGC peak and peak frequency values in the theta range $(4-12 \mathrm{~Hz})$ were compared using paired $t$ test (directionality: HPC $\rightarrow$ RSC vs RSC $\rightarrow$ HPC; state: aWK vs PS) Bonferronicorrected for multiple comparisons.

Phase-amplitude coupling. To quantify the phase-amplitude coupling between different frequency oscillations, a modulation index (MI) was calculated (Tort et al., 2010). After extracting the amplitude time series of a given high-frequency oscillation and the simultaneous phase time series of a given low-frequency oscillation, the MI was defined as the normalized Kullback-Leibler distance of the amplitude distribution across all phases from a uniform distribution. Hippocampal phase and amplitude information were obtained from signals recorded from the pyramidal layer of CA1. Low-frequency oscillations were restricted to theta band range $(4-12 \mathrm{~Hz})$, whereas high-frequency oscillations were subdivided into gamma $(40-100 \mathrm{~Hz})$ and fast gamma $(100-160 \mathrm{~Hz})$ frequency bands. Comodulogram maps were made by calculating the MI between the phase of frequencies ranging from 2 to $20 \mathrm{~Hz}$ ( $1 \mathrm{~Hz}$ bandwidth) and amplitudes ranging from 40 to $250 \mathrm{~Hz}$ ( $5 \mathrm{~Hz}$ bandwidth). No overlap was used for phase or amplitude. LFP data were filtered using a linear finite impulse response filter coded in the eegfilt MATLAB function from the EEGLAB toolbox (Delorme and Makeig, 2004; RRID:SCR_007292). Phases and amplitudes of filtered data were obtained using the Hilbert transform. We used 18 phase equal bins ranging from $-\pi$ to $\pi$ radians to project the distribution of mean amplitudes per bin. Unit firing modulation by brain oscillations was calculated with the same protocol as for phase-amplitude coupling, but using the distribution of the firing probability across slow-oscillation phases instead of the fast-oscillation amplitude (Fig. 5B).

Histology. At the end of the recordings, rats were overdosed with pentobarbital (100 mg/kg, i.p.) and transcardially perfused through the left ventricle with ringer lactate, followed by $4 \%$ paraformaldehyde solution. Brains were removed and stored successively in $4 \%$ paraformaldehyde and 30\% sucrose both for at least $24 \mathrm{~h}$, then frozen, and sectioned in a cryostat (Microm). The final positions of the electrode tips were determined based on a rat atlas (Paxinos and Watson, 1997) after examination of frontal sections counterstained with neutral red (Fig. 1A).

Statistical analysis. Sleep data, LFPs, and unit data were analyzed using one-way ANOVA. Post hoc analyses were conducted using paired and unpaired $t$ tests. Pearson's correlation coefficient $(R)$ was used to determine the linear dependency between two variables. Significance was set at $5 \%$ and Bonferroni correction was applied when multiple comparisons were made. Data are reported as mean \pm SEM.

\section{Results}

\section{Localization of the electrodes in the RSC and ACA}

The position of the electrodes was verified on coronal sections (Fig. 1A). In the RSC, electrodes were localized in the agranular and granular subdivisions, mostly $\sim-5.4 \mathrm{~mm}$ from bregma. In ACA, electrodes were located mostly at $+1.6 \mathrm{~mm}$ anterior to bregma (Fig. 1A). In HPC, electrodes were distributed over CA1, $\mathrm{CA} 3$, and the DG. Analyses were made specifically from the electrodes localized in dorsal CA1. All electrodes were positioned in regions in which Fos-positive neurons were previously observed (Renouard et al., 2015).

\section{Analysis of the LFPs in the HPC, RSC, and ACA}

Recording sessions $(n=23)$ from all animals $(N=12)$ were analyzed during 4 or 8 h between 10:00 A.M. and 6:00 P.M. (total: $147 \mathrm{~h}$ of recording; average $6.39 \pm 0.49 \mathrm{~h}$ /session). Sleep architecture is summarized in Table 1. During aWK (Fig. 1B, left), in contrast to qWK (not illustrated), phasic movements and HPC theta oscillations (Fig. $1 B, C, E, F$ ) were observed. Theta/delta ratio and EMG power were indeed significantly higher during aWK than during qWK (theta/delta ratio: $2.38 \pm 0.62$ vs $1.73 \pm 0.35$; $p<0.05$ and EMG-RMS: $0.068 \pm 0.009$ vs $0.038 \pm 0.007 \mu \mathrm{V}^{2}$; $p<0.001$, for aWK and qWK, respectively). During SWS (NREM sleep), the EEG and LFPs showed mostly delta waves $(0.5-4 \mathrm{~Hz})$ and reduced muscle tone (Fig. $1 B$, middle). Finally, during PS (aka, REM sleep), the ECoG and LFPs showed low-voltage fast activity associated with sustained occurrence of theta oscillation and the complete absence of muscle tone (Fig. 1B, right).

LFPs in HPC, RSC, and ACA showed the same global pattern as the ECoG, during SWS (Fig. 1C). Indeed, quantitative analysis showed that delta $(0.5-4 \mathrm{~Hz})$ and theta $(4-12 \mathrm{~Hz})$ power were not different between structures during SWS (Fig. $1 D, E$ ). In contrast, relative theta power was very high during PS both in HPC and RSC, whereas it was much lower in ACA (Fig. 1C). Quantitative analysis of theta power confirmed that it was significantly increased during PS compared with SWS and aWK in HPC and the RSC but not in ACA (Fig. 1E,F). Theta peak frequency in HPC was also significantly higher during PS $(6.80 \pm 0.09 \mathrm{~Hz})$ than during aWK $(6.4 \pm 0.11 \mathrm{~Hz} ; p<0.02$, paired $t$ test).

\section{Cross-correlation and Granger causality between HPC, RSC, and ACA}

We computed cross-correlations between pairs of raw LFPs recorded in HPC, RSC, and ACA during all vigilance states (Fig. 2A). HPC and RSC showed a strong synchronization in the theta range during PS and to a minor extent during aWK (Fig. 2A, middle). The cross-correlation also revealed the existence of a lower synchronization between ACA and RSC during PS and aWK (Fig. 2A, right), and an even weaker correlation between HPC and ACA (Fig. 2A, left). During SWS, correlations were also observed although no oscillatory pattern emerged. For all pairs, the lag was close to 0 . It was not statistically different between PS and aWK (PS and aWK: HPC-RSC: $-3.92 \pm 3.12 \mathrm{~ms}$ vs $1.33 \pm$ $6.37 \mathrm{~ms}$, HPC-ACA: $9.17 \pm 9.17 \mathrm{~ms}$ vs $-15.17 \pm 18.64 \mathrm{~ms}$, ACA-RSC: $5.67 \pm 19.6 \mathrm{~ms}$ vs $-4.67 \pm 3.83 \mathrm{~ms}$ ). Averaged crosscorrelations including all animals at zero-lag showed significantly increased theta synchronization between HPC and RSC during PS compared with aWK and SWS (Fig. 2B). Other pairs of structures did not show significantly different cross-correlations between behavioral states (Fig. 2B).

We then tested whether the increased synchrony between the HPC and the RSC during PS resulted from enhanced strength and information flow from HPC to RSC or the other way around. We observed significant $(p<0.001$, compared with shuffled data) Granger causality values within the theta range during both aWK and PS states (Fig. 2C). The causality values were significantly higher for $\mathrm{HPC} \rightarrow$ RSC than $\mathrm{RSC} \rightarrow \mathrm{HPC}$, during both aWK (paired $t$ test, $p<0.05$ ) and PS (paired $t$ test, $p<0.02$; Fig. $2 D$ ). Granger causality peak frequency was not different for either directionality (HPC $\rightarrow$ RSC vs RSC $\rightarrow \mathrm{HPC}$ ) and state (aWK vs PS; Fig. 2E).

\section{Phase-amplitude coupling}

To determine whether RSC and ACA activity was influenced by theta recorded in HPC, we analyzed the phase-amplitude coupling between slow $(<20 \mathrm{~Hz}) \mathrm{HPC}$ and fast $(>40 \mathrm{~Hz}) \mathrm{HPC}$, RSC, and ACA oscillations. Because previous data suggested a differential behavior of low- and high-frequency gamma during sleep (Scheffzük et al., 2011), gamma was separated into two frequency bands, named gamma $(40-100 \mathrm{~Hz})$ and fast gamma $(100-160$ $\mathrm{Hz}$; Fig. 3A). Quantitative analysis of the two frequency bands was made during each vigilance state for HPC, RSC, and ACA 
A
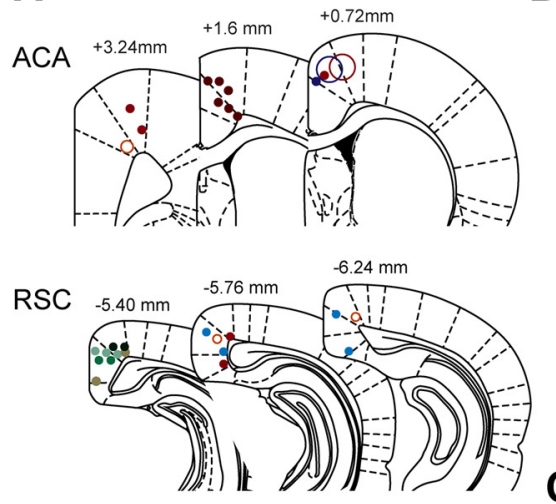

HPC

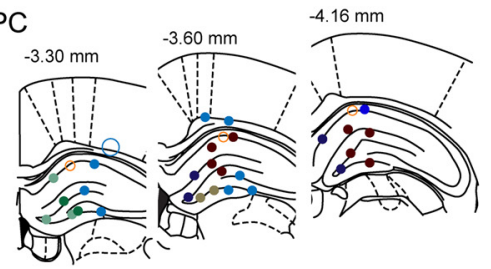

B

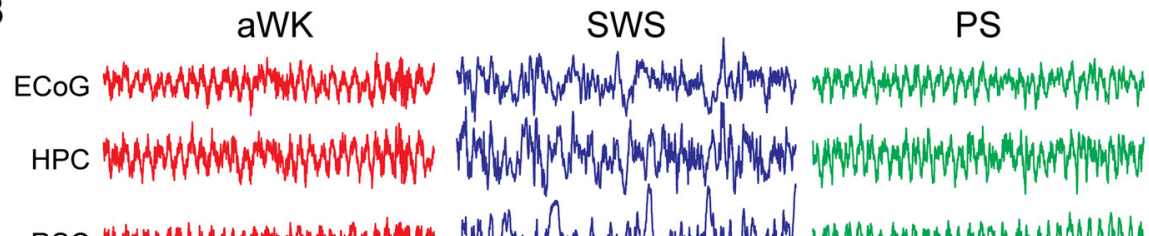

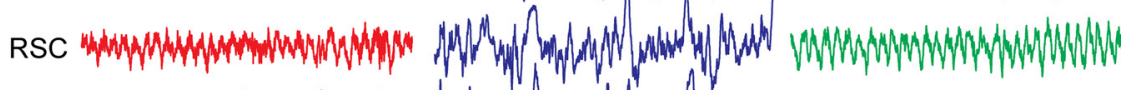

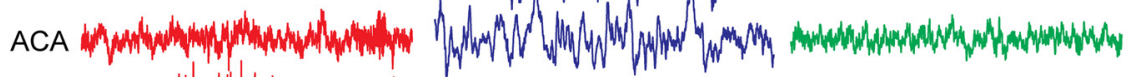
EMG
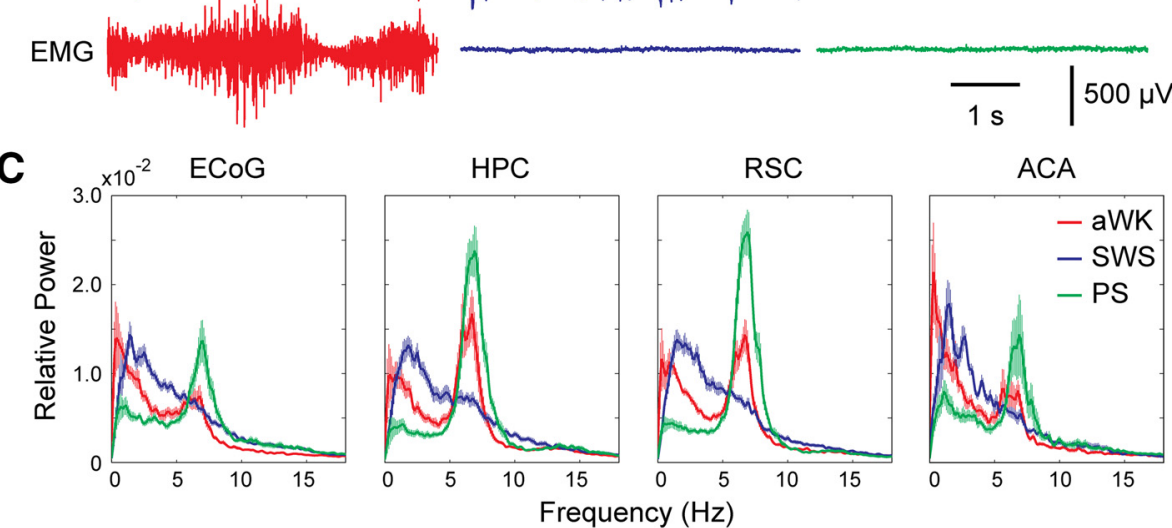

ACA

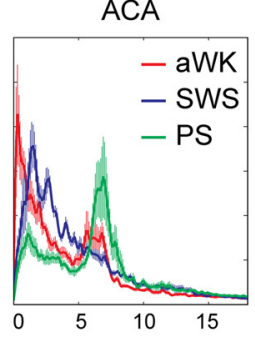

D

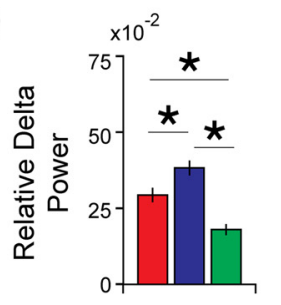

E
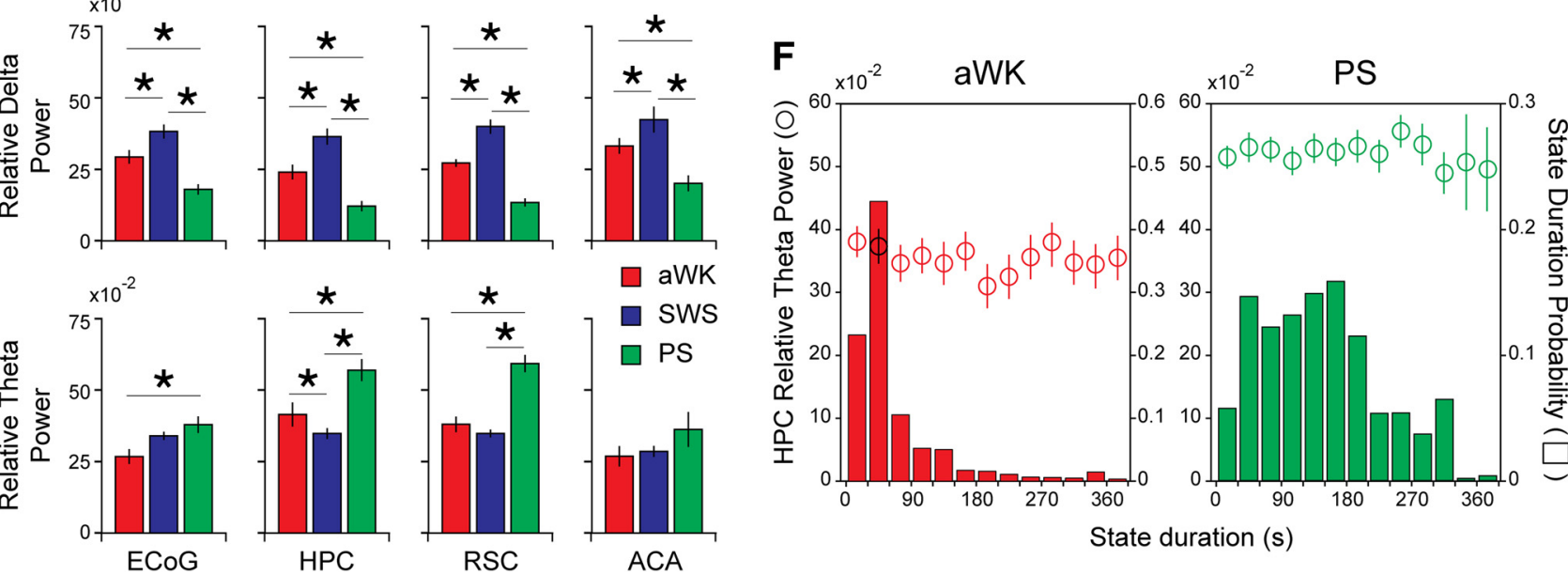

HPC

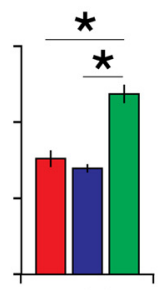

RSC

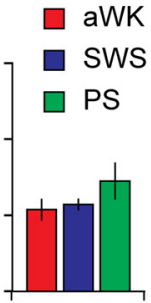

ACA

Figure 1. Analysis of LFPs and ECoG reveals increased theta power in the RSC during PS. A, Schematic coronal sections from the Paxinos and Watson (1997) atlas showing electrode locations in all animals. Anterio-posterior localizations related to bregma are shown above each drawing. $\boldsymbol{B}$, Representative LFPs recorded in HPC, RSC, and ACA during aWK, SWS, and PS. Together with ECoG and EMG, these recordings were used to score the sleep-wake cycle and to generate the hypnograms. C, Grand-average power spectral density for each state in ECOG, HPC, RSC, and ACA. Note the presence of theta oscillation $(\sim 7 \mathrm{~Hz})$ during PS, and to a lesser extent, during aWK. $\boldsymbol{D}, \boldsymbol{E}$, Quantification of delta $(\boldsymbol{D})$ and $(\boldsymbol{E})$ theta power in each structure. Note the significant increase in theta power in HPC and RSC during PS compared with the other states. ${ }^{*} p<0.05$, paired $t$ test. $F$, Grand-average relative theta power during aWK (left) and PS (right) binned according to the state duration (30 s bins). Note that relative theta power is not influenced by state duration.

\section{Table 1. Analysis of the sleep architecture during recordings}

\begin{tabular}{|c|c|c|c|c|c|}
\hline State & aWK & qWk & SWS & IS & PS \\
\hline Time spent in each state, $\%$ & $23.9 \pm 2.8$ & $8.2 \pm 0.9$ & $51.4 \pm 2.3$ & $2.3 \pm 0.2$ & $13.5 \pm 1.0$ \\
\hline No. of episodes & $139 \pm 12$ & $165 \pm 18$ & $133 \pm 12$ & $31 \pm 3$ & $29 \pm 3$ \\
\hline Average episode duration, $s$ & $39.9 \pm 3.3$ & $11.2 \pm 0.6$ & $94.4 \pm 4.5$ & $17.3 \pm 0.6$ & $123.3 \pm 10.0$ \\
\hline Average episode frequency, episode/min & $2.02 \pm 0.16$ & $5.64 \pm 0.28$ & $0.83 \pm 0.03$ & $3.58 \pm 0.13$ & $0.55 \pm 0.03$ \\
\hline No. of persistent episodes, duration $>5$ min & $3.5 \pm 0.5$ & $0.1 \pm 0.1$ & $6.8 \pm 0.9$ & 0 & $0.4 \pm 0.2$ \\
\hline Percentage of "persistent" state, \% & $2.7 \pm 0.4$ & $0.04 \pm 0.03$ & $5.3 \pm 0.7$ & 0 & $5.4 \pm 0.4$ \\
\hline
\end{tabular}

Data represent mean \pm SEM. IS, Intermediate sleep.

(Fig. 3B). No significant difference in the power of the two gamma bands was observed between states in the HPC, except between qWK and aWK (not illustrated). Gamma power in the RSC was significantly higher during PS and aWK compared with SWS and qWK (not illustrated). No significant difference was observed in the RSC for fast gamma between states although it showed the same trend than gamma (Fig. 3B). In ACA, gamma and fast gamma power was not significantly different between vigilance states excepting for fast gamma between aWK and PS (Fig. 3B). Finally, power spectrum analysis showed that frequencies $>160 \mathrm{~Hz}$ were higher in all structures during aWK compared with PS (Fig. 3B). 
A

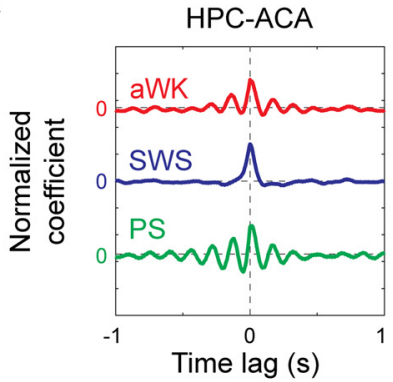

C

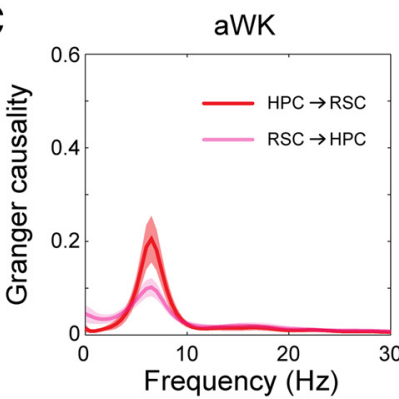

HPC-RSC

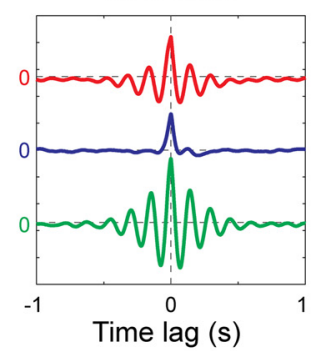

PS

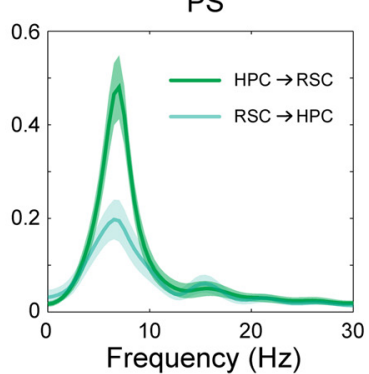

ACA-RSC

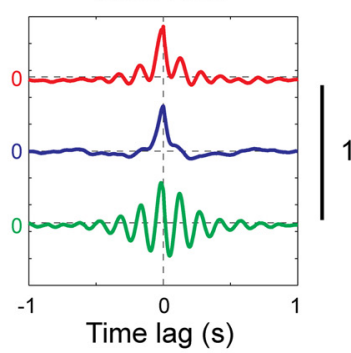

D

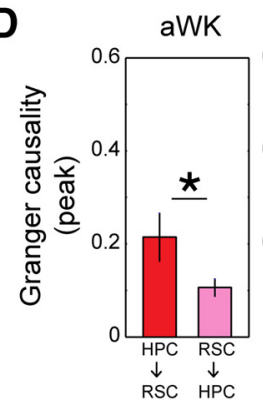

B

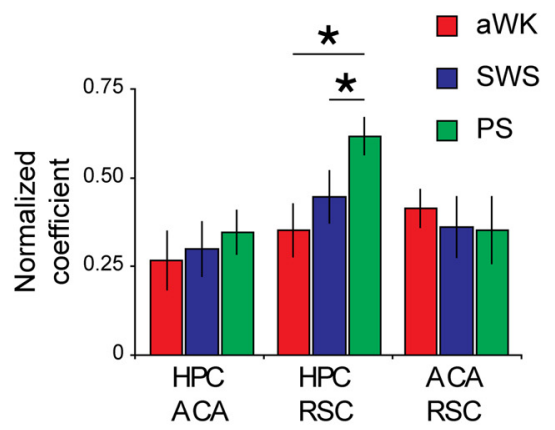

Figure 2. Increased synchrony in the theta frequency band and asymmetrical directionality between HPC-RSC during PS. $A$, Mean normalized cross-correlations between pairs of recordings from HPC, RSC, and ACA during aWK, SWS, and PS. B, Averaged cross-correlation coefficients at lag close to zero shows significantly increased synchronization between HPC and RSC specifically during PS. C, Averaged Granger causality spectra between HPC and RSC during aWK (left) and PS (right) shows significant bidirectional interactions in the theta range. Mean and SEM are represented as thick lines and shaded areas. $\boldsymbol{D}$, Peak Granger causality values and $(\boldsymbol{E})$ peak frequency in the theta frequency range demonstrate stronger interaction in $H P C \rightarrow R S C$ than $R S C \rightarrow H P C$ direction, mainly during PS. ${ }^{*} p<0.05$, paired $t$ test ( $n=6$ for HPC-ACA and ACA-RSC; $n=10$ for HPC-RSC).

Interestingly, an increase in fast gamma power centered on $131 \mathrm{~Hz}$ was clearly visible during PS compared with aWK in the power spectrum of the RSC but not in that of the HPC and ACA (Fig. 3B, arrow). Further, raw and filtered traces suggested that fast gamma activities in RSC and HPC were specifically coupled with HPC theta during PS (Fig. $3 A$ ). We therefore further quantified the coupling between HPC theta phase and HPC and RSC fast oscillations amplitude during the two theta rich states (PS and aWK) in all structures (Fig. $3 C, D$ ). We found that coupling specifically occurred between gamma (centered at $86 \pm 7 \mathrm{~Hz}$ ) and fast gamma (centered at $131 \pm 3 \mathrm{~Hz}$ ) amplitudes and theta (centered at $6.7 \pm 0.1 \mathrm{~Hz}$ ) phase during PS but not during qWK, aWK, and SWS both in HPC and RSC (Fig. 3C). The coupling was similar when calculating it between RSC theta phase and RSC fast oscillations both during PS and aWK (Fig. 3 E). Such coupling did not occur in ACA for any of the vigilance states (Fig. 3C,D). Vigilance states with weak theta power (qWK and SWS) also lacked phase-amplitude coupling in the frequency bands studied (not illustrated). The strength of the coupling, as measured by the MI, was higher for the fast gamma than for gamma (Fig. 3D). The increase in comodulation occurred near the peak of the theta phase in the HPC (Fig. 3F, top) and just after the peak $\left(60^{\circ}\right)$ in the RSC (Fig. 3F, bottom).

Units firing in the ACA and RSC during each vigilance state All electrodes displaying units were localized in the ACA and RSC based on the analysis of their positions in coronal sections (Fig. $1 A)$. Strict criteria were used to isolate the units from background activity such as a signal-to-noise ratio superior to 100 (RSC: $250 \pm 14, n=35$, from 12 rats; ACA: $276 \pm 17, n=23$; from 6 rats) and peak to valley amplitude $>60 \mu \mathrm{V}$ (RSC: $113 \pm 7$; ACA $136 \pm 13 \mu \mathrm{V})$. A hyperplane was used to separate two clusters of waveforms (narrow and wide spikes) in the scatter diagram of the trough-to-peak latency and spike peak asymmetry (Fig. 4C). It has been suggested that repolarization of inhibitory neurons is faster than excitatory ones and the latency between the trough and the following peak could be used in extracellularly recorded units to classify them in two physiologically relevant entities (Sirota et al., 2008). This approach revealed that 18 of 35 and 8 of 23 units from the RSC and ACA, respectively, could be classified as putative inhibitory neurons (PIN, narrow spikes; Fig. 4C). To control for spikes waveform variability across time, we also compared their morphology and amplitude during the entire duration of the recording and found no significant difference (Fig. $4 D$ ). In addition, ISI histograms were used to guarantee that absolute refractory period was always preserved (Fig. $4 B$; average mode of ISI in RSC: $12.4 \pm 2.5$; in ACA: $9.8 \pm 1.4 \mathrm{~ms})$. The normalized and smoothened firing rate profiles of representative RSC and ACA units are shown in Figure $4 E$.

RSC units showed a wide distribution in terms of averaged discharge rate, ranging from 0.06 to $21.5 \mathrm{~Hz}$ (Fig. $4 F$ ). Thus, we normalized their averaged firing to compare their activity across states (Fig. 4G). The normalized firing rate of all individual units was significantly higher during PS (124 $\pm 7 \%)$ and aWK (114 \pm $5 \%$ ) compared with SWS (78 $\pm 3 \%$; Fig. $4 H$ ). To determine whether neurons in a given structure all display the same pattern of activity with regards to the vigilance state, we further examined them individually. We found that 24 of 35 recorded RSC units (68.4\%) showed a significantly higher firing during PS than during any other states. For clarity, these units will be referred as PS+ units (Fig. 4H; Table 2). The spike waveforms of one representative PS+ unit is shown in Figure $4 A$. Among the PS+ units, 14 were of putative inhibitory neurons (PIN) and 10 of putative excitatory neurons (PEN) (putative excitatory neurons, wide spikes in Fig. 4C) types. In addition, 10 RSC units (28.6\%) showed a significantly higher activity during aWK than during the two other states and were thus classified as aWK + . Four of these units were PIN and 6 were PEN. Only one single unit was 
A

aWK

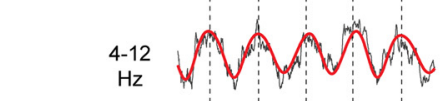

HPC

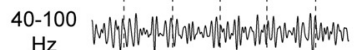
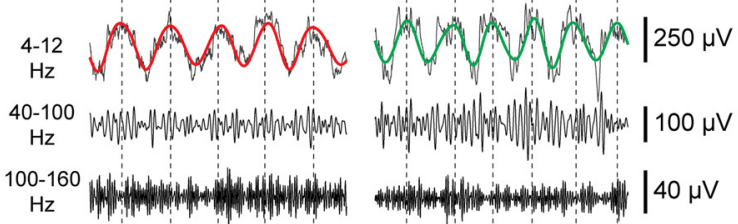

PS

B
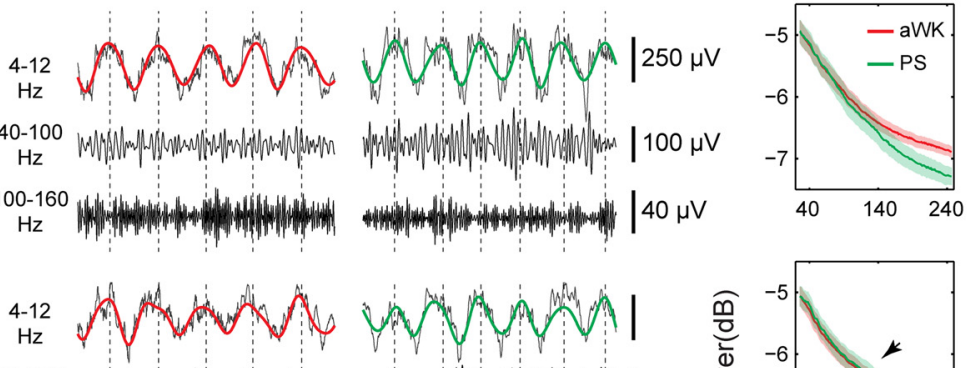

RSC
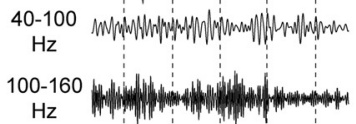

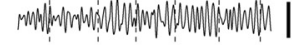

$\mathrm{Hz}$

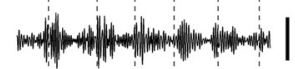

4-12

$\mathrm{Hz}$

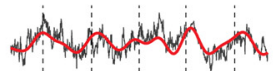

ACA

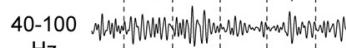
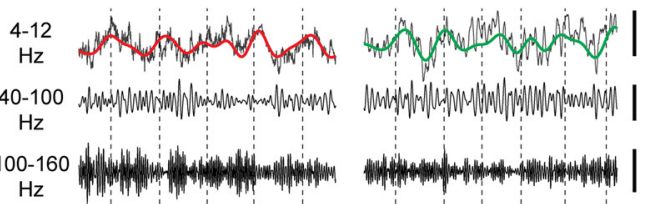

$\overline{200 \mathrm{~ms}}$
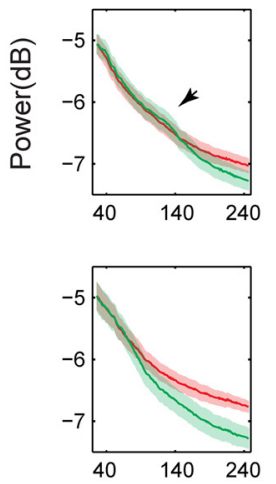

Frequency $(\mathrm{Hz})$
C

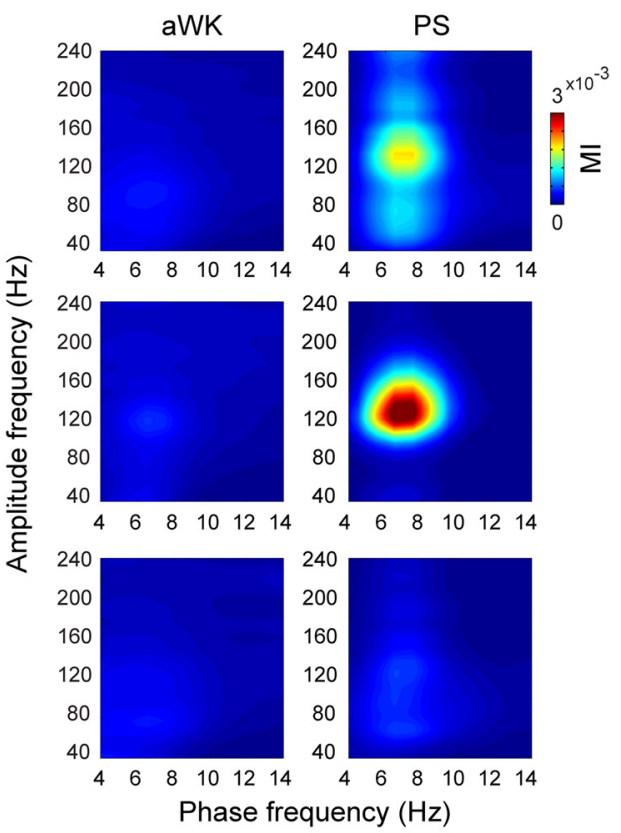

D

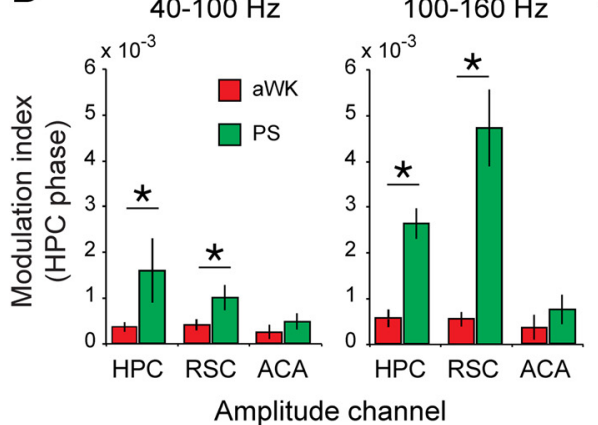

E

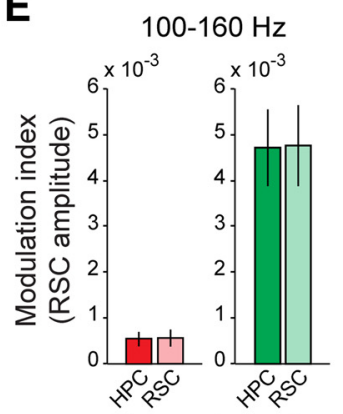

Phase channel
$\mathbf{F}$

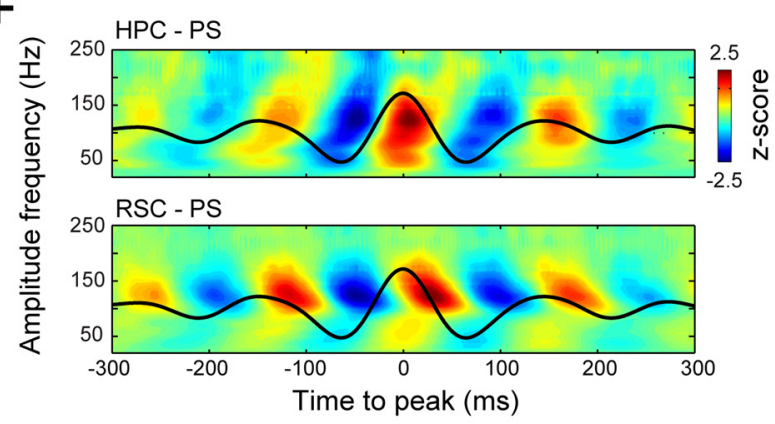

Figure 3. Phase-amplitude coupling (PAC) between hippocampal theta and gamma oscillations in HPC, RSC, and ACA. A, Raw and filtered LFPs recorded in HPC, RSC, and ACA during aWK and PS in one representative animal. $\boldsymbol{B}$, Power spectra of fast oscillations during aWK and PS. C, Pseudocolor-coded averaged cross-frequency coupling maps between hippocampal theta phase and gamma amplitude in HPC, RSC, and ACA during aWK and PS (color bar is set the same for all panels, warm colors indicate strong coupling). D, PAC quantification shows that theta phase modulation of gamma $(40-100 \mathrm{~Hz})$ and fast gamma $(100-160 \mathrm{~Hz})$ is significantly higher during PS than during aWK in HPC and RSC but not in ACA. ${ }^{*} p<0.05$, paired $t$ test. $E$, PAC is similar when calculating it using theta from HPC or RSC. F, Time-frequency plot of averaged gamma amplitude (Z-score) distribution time-locked to the theta peak (0s) in the HPC (top) and RSC (bottom) during PS ( $n=10)$. Hippocampal averaged theta cycles during PS are superimposed on plot traces.

significantly more active during SWS than during the other states (Table 2). Interestingly, PS+ and aWK + unit activity was not positively linked with the EMG root mean square (RMS) during aWK (PS+: $r=0.18 \pm 0.03$; aWK $+: r=0.17 \pm 0.06 ; p>0.05$, Pearson's correlation). Finally, a minority of RSC units (5 PS+ and $2 \mathrm{aWK}+$ ) showed a bimodal ISI histogram with one sharp peak between 2-10 ms, specifically during SWS, suggesting that they display bursts during this state (not illustrated).

We recorded 23 units in the ACA (6 rats) during all vigilance states. As for RSC, these units showed a wide distribution in term of discharge rate, ranging from 0.3 to $28.5 \mathrm{~Hz}$ (Fig. $4 F$ ). The normalized firing rate of these neurons was significantly higher during aWK (117 $\pm 7 \%)$ than SWS (91 $\pm 4 \%)$ but not to PS. No difference was observed between SWS and PS (112 $\pm 8 \%)$. We then classified individually the units based on their firing during each vigilance state. Ten of the ACA units (43.4\%; Table 2) showed a higher activity during PS than during the two other states (PS+ units). Four of these units were PIN and six were PEN. The increased activity of a representative PS + unit during PS compared with the other states is illustrated in Figure 4E. Eight units (35\%) showed a significantly higher activity during aWK than during the two other states (aWK + units; Table 2). One unit was PIN and seven were PEN. As observed for RSC, the PS+ and aWK + ACA units also did not show a positive correlation with the EMG RMS during aWK (PS $+: r=0.21 \pm 0.05$; aWK $+: r=$ $0.20 \pm 0.05 ; p>0.05$, Pearson's correlation). In addition, none of the ACA units showed a bimodal distribution in the ISI histogram during any states suggesting that they do not discharge in bursts (not illustrated). Finally, five ACA units (21.7\%) discharge more during SWS than during the two other states (Table 2).

We next investigated whether RSC and ACA cortical units showed ON and OFF periods of activity in six animals (Fig. 5). OFF periods were defined when all recorded neurons (regardless of their location in the cortex) were silent for at least $50 \mathrm{~ms}$, as described previously (Vyazovskiy et al., 2009). OFF periods mostly occurred during SWS (Fig. 5A,D) and lasted significantly longer compared with those occurring during aWK and PS (Fig. $5 E$ ). The onset of the OFF periods during SWS occurred in phase with the delta waves (Fig. 5B). Interestingly, the duration of the 
A

B
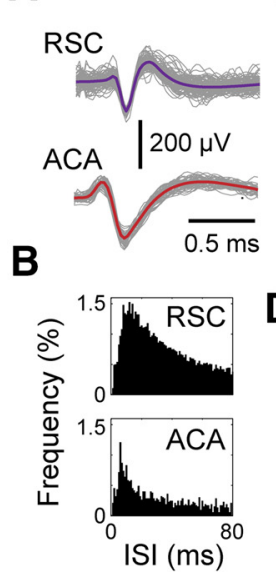

F

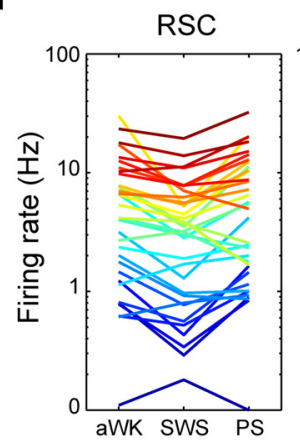

C

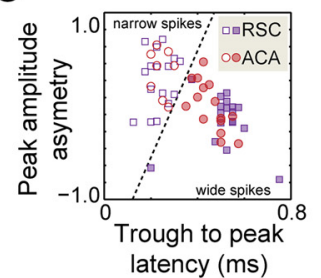

E

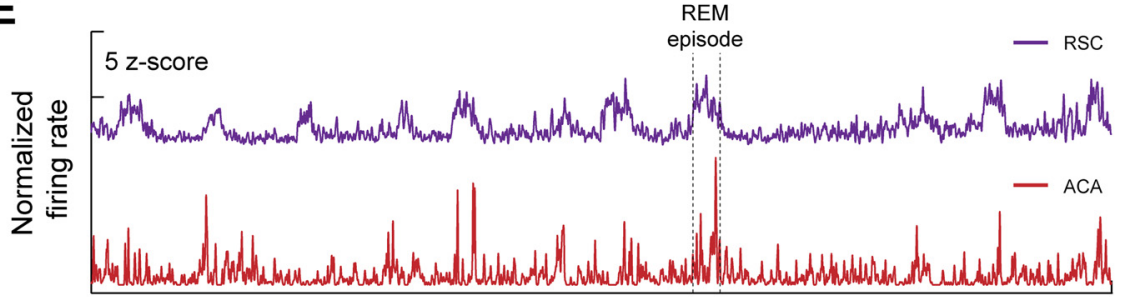

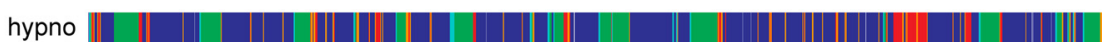

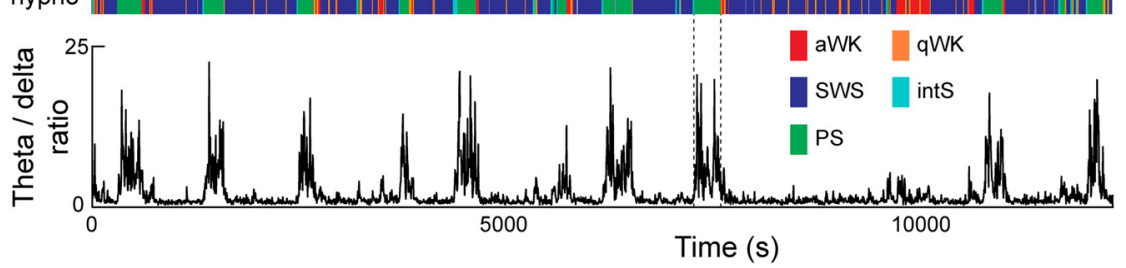

G

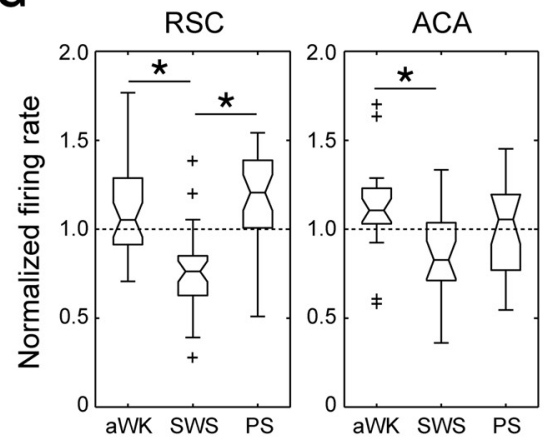

H
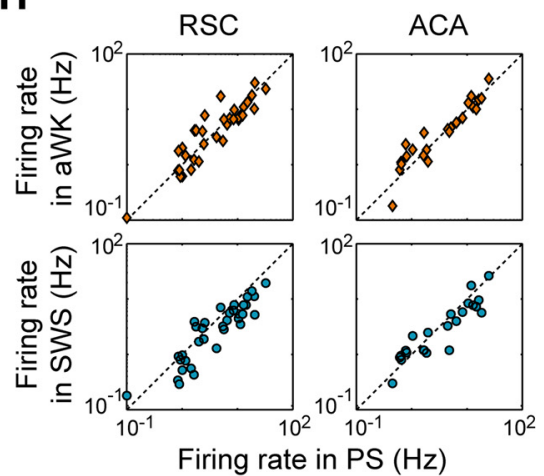

$(\mathrm{Hz})$

Figure 4. ACA and RSC unit activity increases specifically during PS. $A$, Spike waveforms of two representative units in RSC and ACA. B, ISI histograms of the units shown in $A$. C, Spike waveform asymmetry and spike width are calculated to separate the neurons in two classes with narrow (PIN, above the dashed line) and wide (PEN, below the dashed line) spikes. D, Spike amplitude stability over the recording session. $\boldsymbol{E}$, Smoothed spike train of the units shown in $\boldsymbol{A}$ and $\boldsymbol{B}$ convolved with a Gaussian function (STD $=5 \mathrm{~s}$; top) aligned with the hypnogram (middle) and the theta-delta ratio (bottom). Note that both units are more active during PS than the other states, notably the RSC one. $F$, Color-coded mean firing rates of all individual units across vigilance states (colors are defined by SWS mean firing rate, warm colors represent higher firing rates) and (G) normalized population firing rates of all ACA and RSC units during aWK, SWS, and PS. ${ }^{*} p<0.05$, nonparametric paired $t$ test. $\boldsymbol{H}$, Relationship between individual unit firing rates during PS ( $x$-axis), and aWK ( $y$-axis, top), or SWS ( $y$-axis, bottom) in the RSC (left) and ACA (right). Units below the line are more active during PS than aWK or SWS (see Table 2 for further statistical comparisons).

Table 2. Units ranked according to their discharge rate during each vigilance states

\begin{tabular}{|c|c|c|c|c|c|c|}
\hline \multirow[b]{2}{*}{ Units } & \multirow[b]{2}{*}{ State comparison } & \multicolumn{2}{|c|}{$\begin{array}{l}\text { No. of } \\
\text { neurons }\end{array}$} & \multirow[b]{2}{*}{ State comparison } & \multicolumn{2}{|c|}{ Neurons, \% } \\
\hline & & ACA & RSC & & ACA & RSC \\
\hline \multirow[t]{2}{*}{ aWK+ } & $\mathrm{aWK}>\mathrm{SWS}>\mathrm{PS}$ & 5 & 5 & aWK $>S W S>P S$ & 21.7 & 14.3 \\
\hline & aWK $>$ PS $>$ SWS & 3 & 5 & $\mathrm{aWK}>\mathrm{PS}>\mathrm{SWS}$ & 13.0 & 14.3 \\
\hline \multirow[t]{2}{*}{ SWS+ } & $S W S>a W K>P S$ & 3 & 1 & SWS $>$ aWK $>$ PS & 13.0 & 2.9 \\
\hline & $S W S>P S>a W K$ & 2 & 0 & SWS $>$ PS $>$ aWK & 8.7 & 0.0 \\
\hline \multirow[t]{3}{*}{ PS+ } & $P S>a W K>S W S$ & 9 & 20 & $\mathrm{PS}>\mathrm{aWK}>\mathrm{SWS}$ & 39.1 & 57.1 \\
\hline & $P S>S W S>a W K$ & 1 & 4 & $P S>S W S>a W K$ & 4.3 & 11.4 \\
\hline & Sum & 23 & 35 & Total & 100.0 & 97.2 \\
\hline
\end{tabular}

OFF period was positively linked with the amplitude of the delta waves (Fig. 5C).

Phase locking of unit firing with theta

We determined whether ACA and RSC unit activity during aWK and PS showed any relation with hippocampal rhythms by analyzing the phase locking of RSC and ACA units to theta oscillation. Raster plot and raw and filtered LFP traces showing the firing of a representative unit and the theta wave during PS suggested that it is discharging more just after the peak of the theta wave (Fig. 6A). The firing probability distribution of this unit in relation to the theta phase clearly reveals a nonuniform distribu- tion both during aWK and PS (Fig. 6B). The maximum rate is observed $60^{\circ}$ after the theta peak while the minimum rate occurs $60^{\circ}$ after the theta trough. Quantification of the phase preference of aWK + and PS + units, i.e., the phase with the highest firing probability, shows that most RSC units fire after the peak of theta (Fig. 6D). Phase preference of the ACA units is highly variable compared with RSC ones (Fig. $6 D$; phase variance: $26.9^{\circ}$ for RSC and $50.5^{\circ}$ for ACA during PS). The MI significantly increased specifically during PS for the PS + units of the RSC $(p=0.02$; paired $t$ test) but not for those in ACA ( $p=0.65$; paired $t$ test; Fig. 6C).

\section{Discussion}

In the present report, we studied the neuronal activity in HPC, RSC and ACA during the sleep-wake cycle. We showed that theta power was higher during PS than during aWK both in HPC and RSC but not in ACA. Further, cross-correlation analysis revealed a strong synchronization in theta specifically during PS between HPC and RSC. In addition, specific cross-frequency coupling occurs between hippocampal theta phase and gamma during PS in HPC and RSC but not in ACA. In addition, $68 \%$ and $43 \%$ of the units recorded in RSC and ACA show a higher firing rate during PS compared with all other states including aWK, respectively. Finally, we report that RSC but not ACA units discharge 

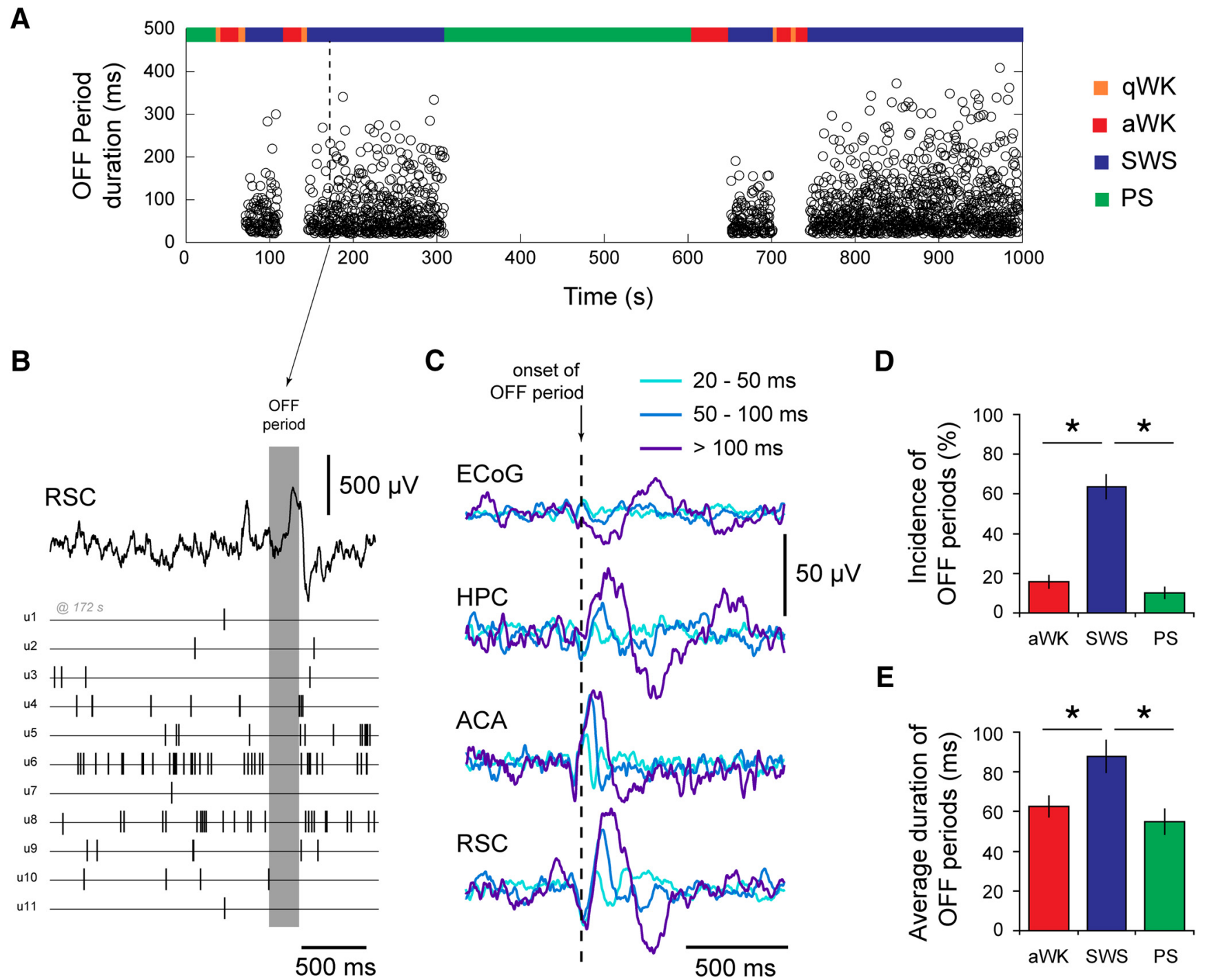

Figure 5. OFF periods occur mainly during SWS. $\boldsymbol{A}$, Hypnogram and dynamics (occurrence and duration) of OFF periods during the different vigilance states. $\boldsymbol{B}$, LFP in the RSC and simultaneously recorded unit activities in the RSC during SWS showing an example of an OFF period with the corresponding delta wave (shaded area). C, Averaged LFP in the RSC signal, locked to the onset of the OFF period (dashed line) sorted by the duration of the OFF period during SWS. $\boldsymbol{D}$, Incidence and $(\boldsymbol{E})$ average duration of OFF periods according to the behavioral state $\left(n=6\right.$ rats). ${ }^{*} p<0.05$, paired $t$ test.

after the peak of HPC theta. Altogether, these results indicate that most RSC neurons show a highly selective activation during PS linked with hippocampal theta oscillation. Below, we discuss our results with regards to previous findings before proposing a functional role of RSC neuronal activation occurring during PS.

Our study constitutes the first report on the activity of the ACA and RSC neurons during the sleep-wake cycle. There is only one previous report showing an increase in theta power in the RSC during PS compared with SWS (Funk et al., 2016). Interestingly, we further report that the increase in theta power during PS in the RSC was similar to that recorded in HPC (our results and Montgomery et al., 2008). In addition, theta was strongly correlated and synchronized between the RSC and HPC as indicated by the cross-correlation analysis. In contrast, theta power was low and not significantly increased in ACA during PS in agreement with a previous study (Sirota et al., 2008). It was also previously reported that theta power monotonically decreases with distance from the HPC and that phase lag increases as a function of distance from the source (Sirota et al., 2008; Lubenov and Siapas,
2009). The theta power decrease observed in the ACA is in line with such observations. In contrast, the presence of theta in the RSC during PS with a power similar to that seen in HPC and the nonzero cross-correlation lag suggests that it might be generated locally (Lachaux et al., 1999). Further, phase locking of units within the RSC suggests that theta oscillation is locally expressed rather than being just volume conducted from the hippocampus. One possibility is that theta recorded in the HPC and the RSC is driven by a common subcortical source. This is very likely because it has been recently shown that medial septum GABAergic neurons generating theta during PS (Borhegyi et al., 2004; Lee et al., 2005; Boyce et al., 2016) directly project to GABAergic interneurons both in CA1 and RSC (Unal et al., 2015). Theta activity in the RSC could also be driven by the GABAergic neurons in the stratum radiatum of CA1, which also projects to RSC interneurons (Miyashita and Rockland, 2007) and fire in burst phase-locked to the descending phase of CA1 pyramidal theta oscillation (Jinno et al., 2007). However, electrolytic HPC lesion reduces theta activity in the RSC but does not affect theta rhythmicity of RSC neurons (Talk et al., 2004). 

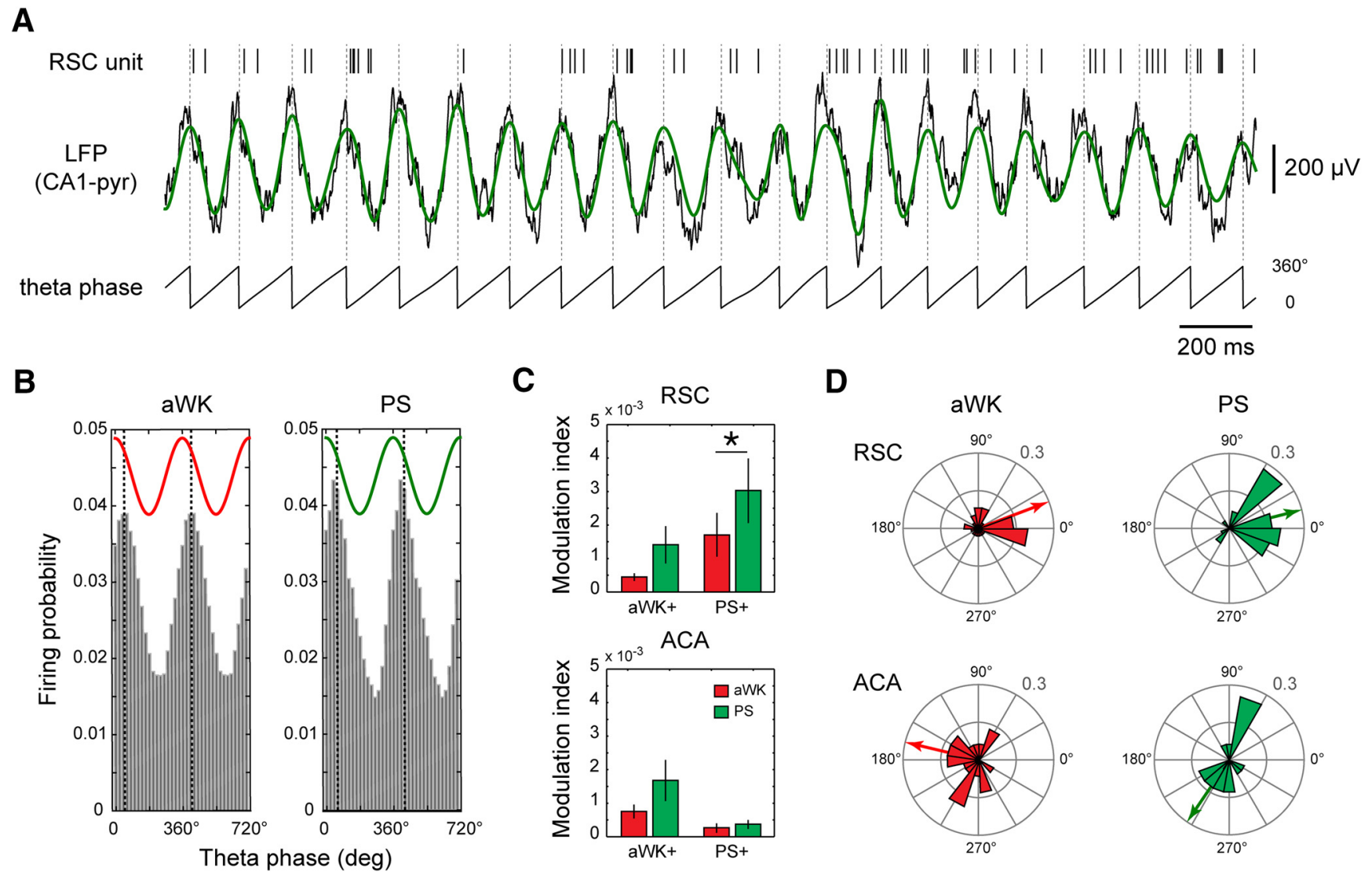

C

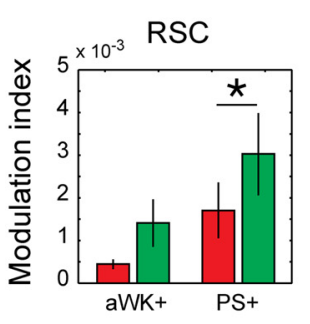

D
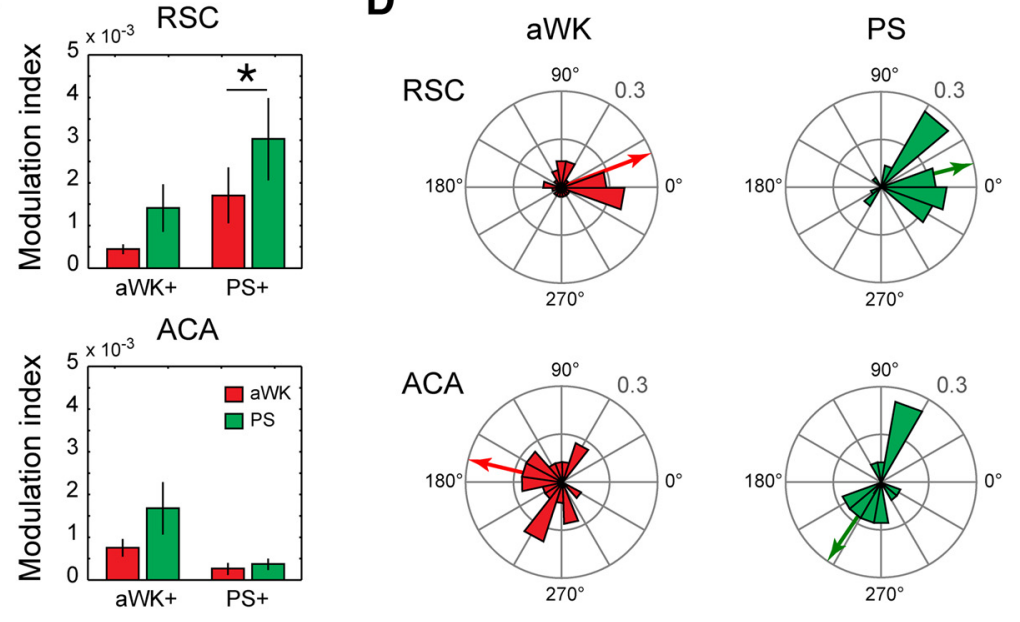

Figure 6. Modulation of units firing by hippocampal theta. $\boldsymbol{A}$, Example of a representative RSC unit firing (raster plot), hippocampal LFP (black: raw trace; green: $4-12$ Hz bandpass filtered), and theta phase during PS. Instantaneous phase of the filtered trace was obtained using Hilbert transformation. $\boldsymbol{B}$, Distribution of the firing probability of the same RSC unit during aWK (left) and PS (right). Dashed vertical lines represent the circular mean of the preferred phase (in this case, $\sim 60^{\circ}$ during aWK and PS). $C$, Mean \pm SEM of the MI for all units in the RSC (top) and ACA (bottom) during aWK and PS. ${ }^{*} p<0.05$, paired $t$ test. D, Polar histogram plot in polar coordinates of the preferred phase for aWK + and PS + units with nonuniform distribution ( $p<0.05$; Rayleigh $z$ test) in RSC (top; $n=30 / 36$ ) and ACA (bottom; $n=16 / 23$ ) during aWK (left) and PS (right). Arrows point to the circular mean of the preferred phase during each state.

We further found that a coupling occurs between theta and gamma during PS and not during aWK in HPC and RSC but not in ACA. Such a result has not been reported before for the RSC. Nevertheless, frequency coupling during PS between HPC theta and neocortical fast gamma was previously reported (Sirota et al., 2008; Scheffzük et al., 2011). Interestingly, HPC-RSC coupling occurred mostly at the same frequency $(131 \mathrm{~Hz})$ than in the superficial layers in the HPC (e.g., pyramidal layer of CA1) rather than at lower frequency $(\sim 80 \mathrm{~Hz})$, as observed at the level of the stratum radiatum/lacunosum-moleculare of CA1 (Sirota et al., 2008; Scheffer-Teixeira et al., 2012). This observation suggests that theta fast-gamma coupling may share similar mechanisms in the HPC (principal layer of CA1) and the RSC, which might be important for information processing and integration across multiple distant networks (Womelsdorf et al., 2007; Canolty and Knight, 2010).

In addition, we found that $68 \%$ of the RSC and $43 \%$ of the ACA units show a significantly higher firing rates during PS compared with all other states. Most of the other units recorded in the two structures were more active during aWK than the other states. To our knowledge, our results constitute the first report showing that a neocortical structure contains a majority of neurons significantly more active during PS than during the other states. Indeed, previous unit recordings of neurons located in the somatosensory cortices showed that the discharge rate of neocortical neurons is similar during PS and aWK, and higher than during SWS (Steriade et al., 2001; Vyazovskiy et al., 2009). These results as well as ours are in line with our previous findings that the RSC and to a minor extent the ACA contain a large number of Fos-labeled cells after PS hypersomnia, in contrast to the somatosensory cortices containing only a few neurons (Renouard et al., 2015). They corroborate the indirect demonstration using Fos that these two structures and, in particular the RSC, contain neurons specifically and strongly active during PS. Such high activity in the RSC and the ACA contrasts with recent reports showing the presence of slow waves (Funk et al., 2016) and decreased unit activity in the somatosensory cortices during PS compared with aWK (Niethard et al., 2016). We further showed that $83 \%$ of the RSC but none of the ACA neurons mostly discharge after the peak of theta. Interestingly, such coupling has been previously described during PS for only $5-40 \%$ of the neurons recorded in the primary somatosensory and prefrontal cortices (Sirota et al., 2008). In contrast, $80 \%$ of the pyramidal cells and $90 \%$ of the interneurons in CA1 are significantly phase-locked to theta oscillations (Csicsvari et al., 1999). Therefore, our results indicate that the synchrony of neurons with theta during PS is as strong in the RSC as in the HPC. In summary, our results indicate for the first time that the RSC contains neurons specifically active during PS phase-locked with theta oscillations.

What could be the function of the activation of RSC and ACA neurons during PS? Both cortices have been involved in spatial learning in rodents (Cho and Sharp, 2001; Frankland et al., 2004; 
Katche et al., 2013) and in the genesis and recall of complex and coherent scenes or events in humans (Maddock, 1999). It has also been shown that human RSC display transient theta highfrequency oscillation coupling during autobiographical memory retrieval (Foster et al., 2013). Further, RSC and the ACA are densely interconnected with the majority of cortical and subcortical brain structures involved in spatial navigation such as the HPC, medial entorhinal cortex, subiculum, and anterior thalamic nuclei (Wyss and Van Groen, 1992). Humans with RSC damage also show impairment in navigational and episodic memory (Maeshima et al., 2001). Lesion and inactivation experiments in rats and mice confirmed that the RSC and ACA play a role in spatial learning (Frankland et al., 2004; Czajkowski et al., 2014). The firing properties of RSC neurons in behaving animals are also consistent with such hypothesis. Indeed, subpopulations of RSC neurons exhibits head-direction tuning preferences (Cho and Sharp, 2001) and map the conjunction of internal and external spaces (Alexander and Nitz, 2015). In view of all these and our results, the activation of RSC neurons during PS and their phaselocked activity to theta oscillation could be involved in contextual memory consolidation. Supporting such hypothesis, LTP and LTD, classical electrophysiological correlates of memory, are preferentially elicited by stimulation during the peak and the trough of an ongoing theta oscillation, respectively (Hyman et al., 2003). Moreover, optogenetic inhibition during PS of GABAergic neurons in the medial septum projecting to the HPC and RSC, strongly decreased theta power and induced contextual memory impairment (Boyce et al., 2016). To confirm such a hypothesis, it is now necessary to determine whether RSC neurons are activated both during learning tasks and subsequent PS episodes. It remains also to be shown that their inactivation specifically during PS impairs learning consolidation. Finally, to the extent that the vividness of oneiric images implies the existence of neocortical neurons highly active during PS, dreaming is another phenomenon possibly related to the activity of RSC PS + neurons.

In summary, our results indicate that a substantial number of ACA and the majority of RSC neurons are strongly and specifically activated during PS and tightly linked to theta rhythm. Such activation may play a significant role in the consolidation of spatial and emotional memories and in the generation of dreams. Additional experiments are needed to test these hypotheses.

\section{References}

Alexander AS, Nitz DA (2015) Retrosplenial cortex maps the conjunction of internal and external spaces. Nat Neurosci 18:1143-1151. CrossRef Medline

Barnett L, Seth AK (2014) The MVGC multivariate Granger causality toolbox: a new approach to Granger-causal inference. J Neurosci Methods 223:50 - 68. CrossRef Medline

Boissard R, Gervasoni D, Schmidt MH, Barbagli B, Fort P, Luppi PH (2002) The rat ponto-medullary network responsible for paradoxical sleep onset and maintenance: a combined microinjection and functional neuroanatomical study. Eur J Neurosci 16:1959-1973. CrossRef Medline

Borhegyi Z, Varga V, Szilágyi N, Fabo D, Freund TF (2004) Phase segregation of medial septal GABAergic neurons during hippocampal theta activity. J Neurosci 24:8470-8479. CrossRef Medline

Boyce R, Glasgow SD, Williams S, Adamantidis A (2016) Causal evidence for the role of REM sleep theta rhythm in contextual memory consolidation. Science 352:812-816. CrossRef Medline

Bressler SL, Seth AK (2011) Wiener-Granger causality: a well established methodology. Neuroimage 58:323-329. CrossRef Medline

Canolty RT, Knight RT (2010) The functional role of cross-frequency coupling. Trends Cogn Sci 14:506-515. CrossRef Medline

Cho J, Sharp PE (2001) Head direction, place, and movement correlates for cells in the rat retrosplenial cortex. Behav Neurosci 115:3-25. CrossRef Medline
Csicsvari J, Hirase H, Czurkó A, Mamiya A, Buzsáki G (1999) Oscillatory coupling of hippocampal pyramidal cells and interneurons in the behaving rat. J Neurosci 19:274-287. Medline

Czajkowski R, Jayaprakash B, Wiltgen B, Rogerson T, Guzman-Karlsson MC, Barth AL, Trachtenberg JT, Silva AJ (2014) Encoding and storage of spatial information in the retrosplenial cortex. Proc Natl Acad Sci U S A 111:8661-8666. CrossRef Medline

Delorme A, Makeig S (2004) EEGLAB: an open source toolbox for analysis of single-trial EEG dynamics including independent component analysis. J Neurosci Methods 134:9-21. CrossRef Medline

Foster BL, Kaveh A, Dastjerdi M, Miller KJ, Parvizi J (2013) Human retrosplenial cortex displays transient theta phase locking with medial temporal cortex prior to activation during autobiographical memory retrieval. J Neurosci 33:10439-10446. CrossRef Medline

Frankland PW, Bontempi B, Talton LE, Kaczmarek L, Silva AJ (2004) The involvement of the anterior cingulate cortex in remote contextual fear memory. Science 304:881-883. CrossRef Medline

Funk CM, Honjoh S, Rodriguez AV, Cirelli C, Tononi G (2016) Local slow waves in superficial layers of primary cortical areas during REM sleep. Curr Biol 26:396-403. CrossRef Medline

Hyman JM, Wyble BP, Goyal V, Rossi CA, Hasselmo ME (2003) Stimulation in hippocampal region CA1 in behaving rats yields long-term potentiation when delivered to the peak of theta and long-term depression when delivered to the trough. J Neurosci 23:11725-11731. Medline

Jinno S, Klausberger T, Marton LF, Dalezios Y, Roberts JD, Fuentealba P, Bushong EA, Henze D, Buzsáki G, Somogyi P (2007) Neuronal diversity in GABAergic long-range projections from the hippocampus. J Neurosci 27:8790-8804. CrossRef Medline

Jouvet M, Michel F (1959) Corrélations électromyographiques du sommeil chez le chat décortiqué et mésencéphalique chronique. C R Seances Soc Biol Fil 153:422-425. Medline

Katche C, Dorman G, Slipczuk L, Cammarota M, Medina JH (2013) Functional integrity of the retrosplenial cortex is essential for rapid consolidation and recall of fear memory. Learn Mem 20:170-173. CrossRef Medline

Lachaux JP, Rodriguez E, Martinerie J, Varela FJ (1999) Measuring phase synchrony in brain signals. Hum Brain Mapp 8:194-208. CrossRef Medline

Lee MG, Hassani OK, Alonso A, Jones BE (2005) Cholinergic basal forebrain neurons burst with theta during waking and paradoxical sleep. J Neurosci 25:4365-4369. CrossRef Medline

Lubenov EV, Siapas AG (2009) Hippocampal theta oscillations are travelling waves. Nature 459:534-539. CrossRef Medline

Maddock RJ (1999) The retrosplenial cortex and emotion: new insights from functional neuroimaging of the human brain. Trends Neurosci 22: 310-316. CrossRef Medline

Maeshima S, Ozaki F, Masuo O, Yamaga H, Okita R, Moriwaki H (2001) Memory impairment and spatial disorientation following a left retrosplenial lesion. J Clin Neurosci 8:450-451. CrossRef Medline

Maloney KJ, Cape EG, Gotman J, Jones BE (1997) High-frequency gamma electroencephalogram activity in association with sleep-wake states and spontaneous behaviors in the rat. Neuroscience 76:541-555. CrossRef Medline

Miyashita T, Rockland KS (2007) GABAergic projections from the hippocampus to the retrosplenial cortex in the rat. Eur J Neurosci 26:11931204. CrossRef Medline

Montgomery SM, Sirota A, Buzsáki G (2008) Theta and gamma coordination of hippocampal networks during waking and rapid eye movement sleep. J Neurosci 28:6731-6741. CrossRef Medline

Niethard N, Hasegawa M, Itokazu T, Oyanedel CN, Born J, Sato TR (2016) Sleep-stage-specific regulation of cortical excitation and inhibition. Curr Biol 26:2739-2749. CrossRef Medline

Paxinos G, Watson C (1997) The rat brain in stereotaxic coordinates, Ed 3. Sydney; Orlando: Academic.

Renouard L, Billwiller F, Ogawa K, Clément O, Camargo N, Abdelkarim M, Gay N, Scoté-Blachon C, Touré R, Libourel PA, Ravassard P, Salvert D, Peyron C, Claustrat B, Léger L, Salin P, Malleret G, Fort P, Luppi PH (2015) The supramammillary nucleus and the claustrum activate the cortex during REM sleep. Sci Adv 1:e1400177. CrossRef Medline

Scheffer-Teixeira R, Belchior H, Caixeta FV, Souza BC, Ribeiro S, Tort AB (2012) Theta phase modulates multiple layer-specific oscillations in the CA1 region. Cereb Cortex 22:2404-2414. CrossRef Medline 
Scheffzük C, Kukushka VI, Vyssotski AL, Draguhn A, Tort AB, Brankačk J (2011) Selective coupling between theta phase and neocortical fast gamma oscillations during REM-sleep in mice. PLoS One 6:e28489. CrossRef Medline

Sirota A, Montgomery S, Fujisawa S, Isomura Y, Zugaro M, Buzsáki G (2008) Entrainment of neocortical neurons and gamma oscillations by the hippocampal theta rhythm. Neuron 60:683-697. CrossRef Medline

Steriade M, Timofeev I, Grenier F (2001) Natural waking and sleep states: a view from inside neocortical neurons. J Neurophysiol 85:1969-1985. Medline

Talk A, Kang E, Gabriel M (2004) Independent generation of theta rhythm in the hippocampus and posterior cingulate cortex. Brain Res 1015:15-24. CrossRef Medline

Tort AB, Komorowski R, Eichenbaum H, Kopell N (2010) Measuring phase-amplitude coupling between neuronal oscillations of different frequencies. J Neurophysiol 104:1195-1210. CrossRef Medline

Unal G, Joshi A, Viney TJ, Kis V, Somogyi P (2015) Synaptic targets of medial septal projections in the hippocampus and extrahippocampal cortices of the mouse. J Neurosci 35:15812-15826. CrossRef Medline

Vyazovskiy VV, Olcese U, Lazimy YM, Faraguna U, Esser SK, Williams JC, Cirelli C, Tononi G (2009) Cortical firing and sleep homeostasis. Neuron 63:865-878. CrossRef Medline

Womelsdorf T, Schoffelen JM, Oostenveld R, Singer W, Desimone R, Engel AK, Fries P (2007) Modulation of neuronal interactions through neuronal synchronization. Science 316:1609-1612. CrossRef Medline

Wyss JM, Van Groen T (1992) Connections between the retrosplenial cortex and the hippocampal formation in the rat: a review. Hippocampus 2:1-11. CrossRef Medline 